\title{
Die pertrochantäre Femurfraktur
}

\author{
Extramedulläre Fixation mittels dynamischer Hüftschraube (DHS)
}

Dietrich Schulte-Bockholt, Paul Alfred Grützner, Alfred Schmidgen, Andreas Wentzensen

\section{Zusammenfassung}

Die DHS ist auch heute noch ein bewährtes und kostengünstiges Osteosyntheseverfahren insbesondere für die A1-Frakturen. Die Operationstechnik ist standardisiert und weist bei korrekter Indikationsstellung eine geringe Komplikationsrate auf. Die Indikation für die A2/A3-Frakturen ist jedoch kritisch zu sehen. Diese Frakturtypen sind durch die Wahl eines intramedullären Kraftträgers wie des PFN oder Gammanagels stabiler versorgt.

\section{Einleitung}

Die pertrochantäre Fraktur des Femurs ist einer der häufigsten Knochenbrüche des alten Menschen. Sie tritt 4-mal so häufig auf wie die Schenkelhalsfraktur, und die Patienten sind im Schnitt 10 bis 12 Jahre älter als Patienten mit Schenkelhalsfrakturen. Sie verläuft immer extrakapsulär, so dass hier in der Regel nicht wie bei der benachbarten Schenkelhalsfraktur die Gefahr der Durchblutungsstörung des Hüftkopfes mit daraus resultierender Hüftkopfnekrose besteht. Des Weiteren ist der Knochen in der Trochanterregion spongiös, was einer Knochenbruchheilung entgegen kommt. Als Optionen stehen intra- und extramedulläre Stabilisierungsverfahren zur Verfügung. Auch die primäre Endoprothese hat in bestimmten Situationen ihren Stellenwert, während die früher häufig durchgeführte Bündelnagelung nach Ender aufgrund der mangelnden Primärstabilität der Osteosynthese praktisch keine Bedeutung mehr

OP-JOURNAL 2002; 17: 122-127

(C) Georg Thieme Verlag Stuttgart - New York hat. Obwohl die modernen intramedullären Verfahren biomechanisch günstiger sind (LIT), hat die DHS nach wie vor eine Berechtigung in der modernen Osteosynthese bei bestimmten Frakturtypen. Im Folgenden werden die uns sinnvoll erscheinenden Indikationen und die Implantationstechnik der bewährten Osteosynthese dargestellt.

Die klinische Diagnose der pertrochantären Femurfraktur ist meist einfach. In der Regel handelt es sich um eine Monoverletzung deren Ursache ein direkter Sturz auf die Hüfte ist. Es findet sich eine Beinverkürzung in Verbindung mit einer Außenrotation und der Unfähigkeit das Bein zu belasten. Röntgenaufnahmen (Abb.1) des Hüftgelenkes in 2 Ebenen sichern die Diagnose und erlauben eine Klassifikation.

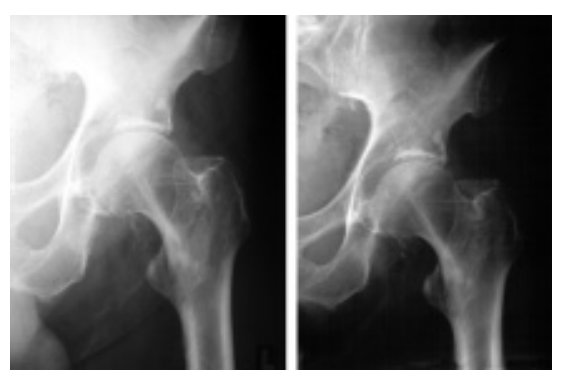

Abb.1 Pertrochantere Femurfraktur.

Zur Einteilung der Frakturen ist die AO Klassifikation (Abb.2) die heute gebräuchlichste Form. Die Brüche werden unterteilt von 31-A1 bis A3 je nach Frakturverlauf, woraus sich dann auch die Einteilung in stabile und instabile Brüche ergibt und letztendlich auch in leicht oder schwer operativ zu versorgende Brüche.

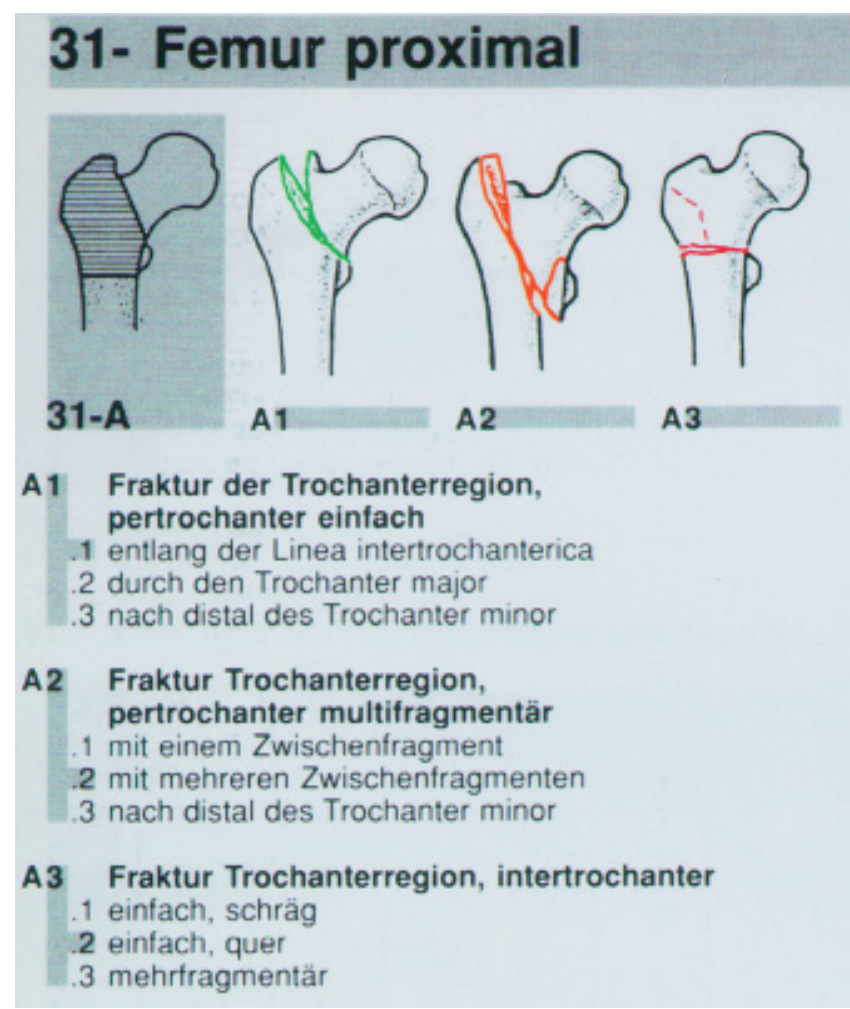

Abb. 2 Klassifikation nach $\mathrm{AO}$. 

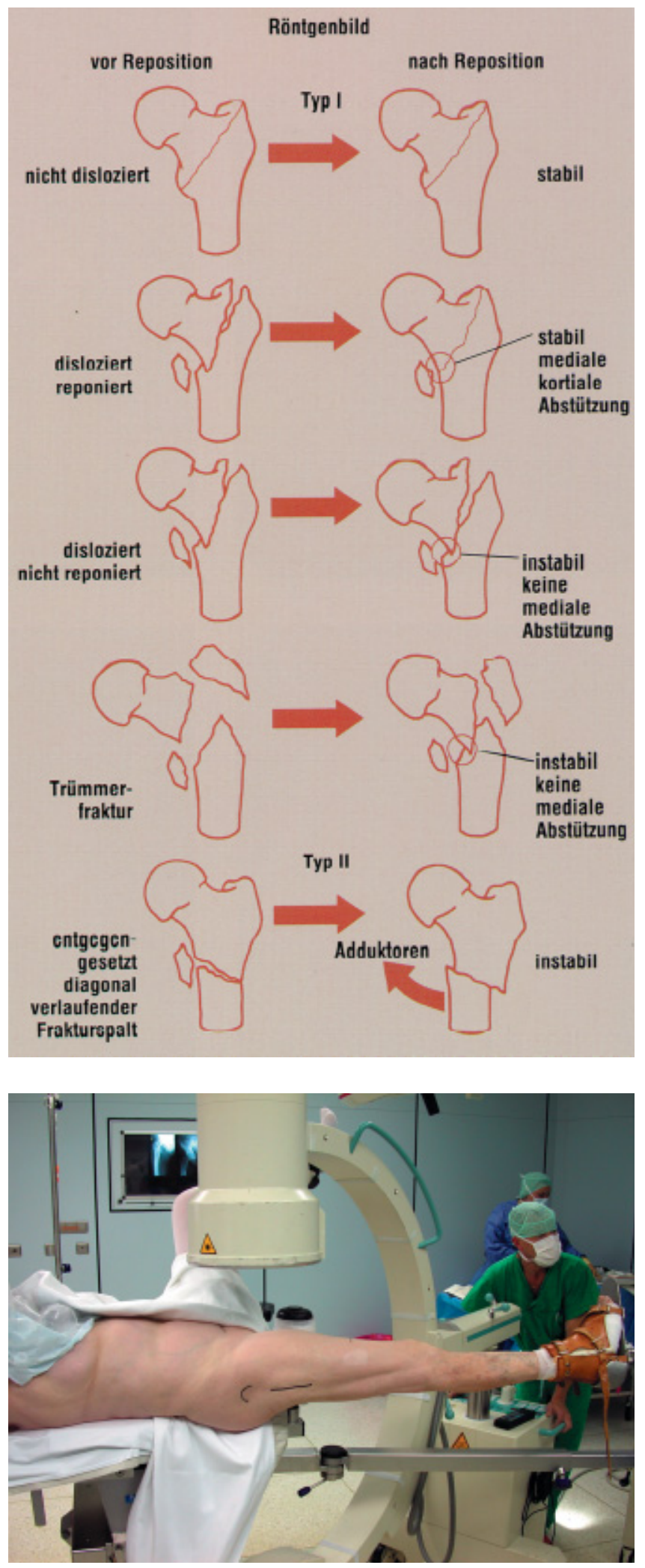

Abb. 3 Klassifikation nach Evan.

AO Klassifikation

31-A1 = einfache Fraktur gute mediale Abstützung.

31-A2 = Mehrfragmentfraktur mediale Abstützung fehlt, Trochanter minor ausgebrochen.

31-A3 = intertrochantäre Fraktur, Fraktur mit subtrochantärem Verlauf, reversed fracture.

Im angelsächsischen Sprachraum ist auch die Klassifikation nach Evan verbreitet (Abb. 3).

Die operative Behandlung der pertrochantären Frakturen ist allgemein anerkannt, da die konservative Behandlung eine ca. 4-6-wöchige Immobilisation notwendig macht, die für den älteren $\mathrm{Pa}-$ tienten, mit einer hohen Mortalität belegt ist.

Ziel der Behandlung ist es, dem Patienten den präoperativen Aktivitätsgrad schnell wieder zu ermöglichen. Günstigster Operationszeitpunkt sind die ersten 24 Stunden. Die perioperative Mortalität nimmt 48 Stunden nach Aufnahme um das $10-$ fache zu. Die Wiederherstellung der regelrechten Anatomie wird angestrebt. Eine anatomische Reposition ist jedoch bei einer ausgeprägten Trümmerzone nicht immer möglich.

Die Stabilität ist abhängig von:

- Frakturverlauf

- Knochenqualität

- Art und Form der Osteosynthese

- Repositionsergebnis

Abb. 4 Lagerung des Patienten auf Extensionstisch.

\section{Anforderung an das Implantat}

Das Implantat soll das Abkippen der Fraktur in mediale (Varusstellung) und dorsale Richtung verhindern und eine stabile Fixation der Fragmente unter Kompression bis zur Frakturheilung ermöglichen. Ziel ist vor allem beim älteren Menschen ein möglichst rasches Erlangen der vollen Belastbarkeit des Beines.

Zur Behandlung der pertrochantären Femurfraktur stehen prinzipiell 2 Verfahren zur Verfügung.:

- intramedulläre Verfahren: PFN oder Gammanagel

- extramedulläre Verfahren: DHS, DCS oder die $95^{\circ}$ Winkelplatte.

Aufgrund der biomechanischen Vorteile der intramedullären Kraftträger bei instabilen Frakturen (A2/A3) setzen wir hier bevorzugt den PFN ein. Bei stabilen Frakturen (A1) bevorzugen wir, aufgrund des komplikationsarmen, bewährten und 


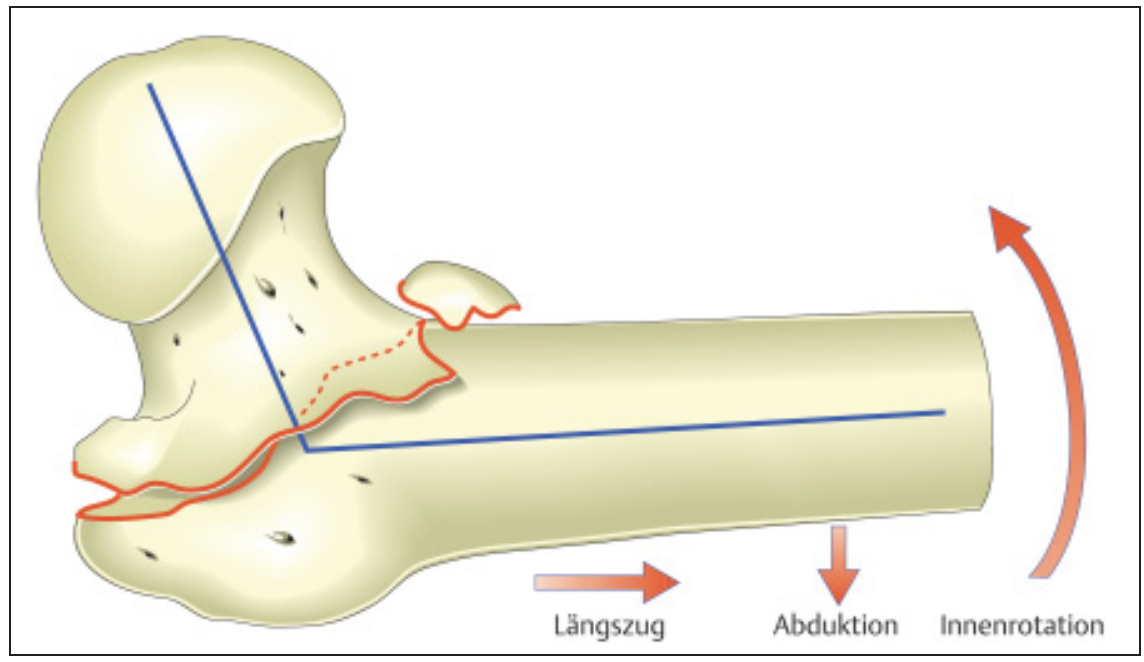

Abb. 5 Schema des Repositionsmanövers.

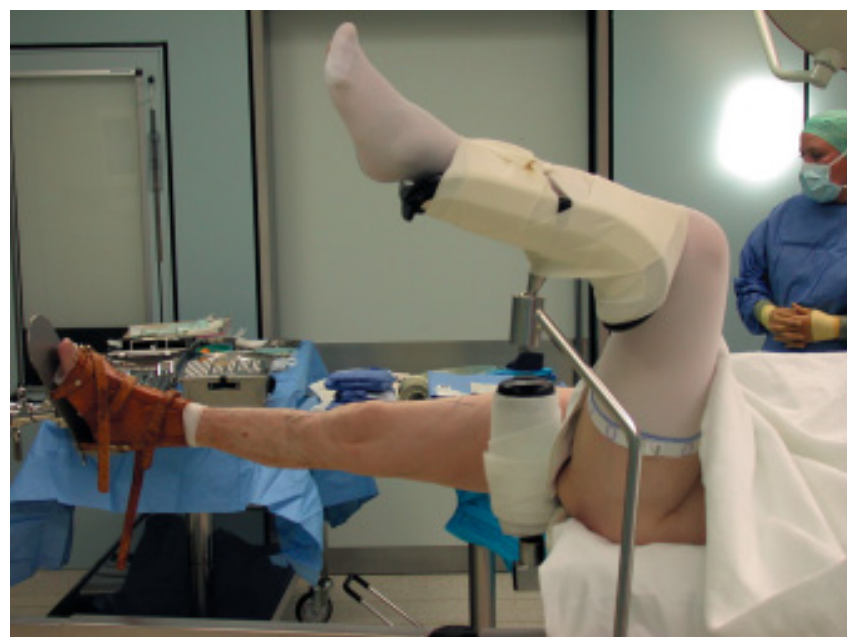

Abb. 6 Lagerung des unverletzten Beines auf dem Extensionstisch.

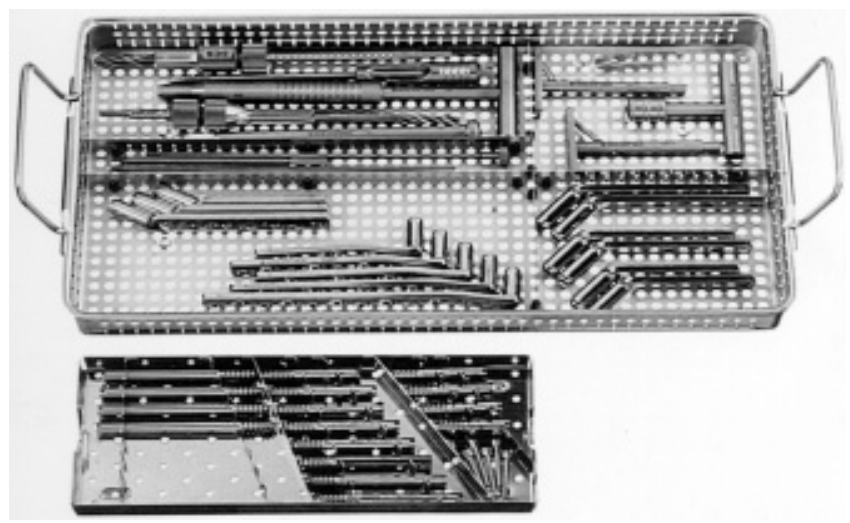

Abb. 7 StandardDHS-Sieb.

operativ einfachen Verfahrens, mit Möglichkeit der Fragmentkompression und der frühfunktionellen Behandlungsmöglichkeit unter Vollbelastung, die DHS. Die DHS ist hier gegenüber dem PFN das deutlich wirtschaftlichere Implantat.

\section{Operation}

Im Operationssaal wird der Patient in Rückenlage in der Regel auf dem Extensionstisch gelagert (Abb.4).

Geschlossene exakte Reposition der Fraktur durch Innenrotation, Längszug und Abduktion unter BV-Kontrolle (Abb. 5).

Das unverletzte Bein wird im Goebelhalter ausgelagert (Abb. 6).

Die Abdeckung erfolgt mittels selbstklebendem U- und Anästhesietuch, das ausgelagerte Bein wird mittels Sack und Beintuch abgedeckt. Alternativ kann auch ein selbstklebendes, durchsichtiges Abdeck-Set (Vertikaltuch) verwandt werden. Der Bildwandler ist zur Operation steril bezogen.

\section{Zugang}

Längsschnitt vom Trochanter major nach distal ca. 15-20 cm.

Längsspalten der Fascia lata in Faserrichtung. Darstellen des proximalen Femurschaftes unter Ablösung der lateralen Vastusmuskulatur von der Septum intermusculare laterale.

Einschieben eines Kirschner-Drahtes über den Schenkelhals ventralseitig zur Bestimmung des CCD-Winkel und der Antetorsion des Schenkelhalses.

Bei instabilen Frakturen kann das Repositionsergebnis durch temporär eingebrachte Kirschner-Drähte optimiert werden.

Planung der Schraubenlage und Auswahl der notwendigen DHS-Platte (zur Verfügung stehen $135^{\circ}-, 140^{\circ}-, 145^{\circ}-$ und $150^{\circ}$-Platte) entsprechend des Schenkelhals- Femurschaftwinkels (Abb. 7).

Aufsetzen des Zielgerätes und korrekte Platzierung des Führungsdrahtes mit Gewinde im subchondralen Knochen.

Der Führungsdraht liegt idealerweise $6 \mathrm{~mm}$ kranial des Adamschen Bogens im dorsokaudalen Quadranten (Abb. 8).

Überprüfung der Lage des Führungsdrahtes sowohl im a.p. als auch axialen Strahlengang und ggf. Korrektur.

Die exakte Platzierung des Führungsdrahtes ist die Voraussetzung für eine erfolgreiche Operation.
Falls die operative Versorgung sofort möglich ist, wird das Bein auf dem Weg in den OP schmerzfrei in einer Schaumstoffschiene gelagert. Ist eine sofortige Operation in Ausnahmefällen nicht möglich, wird eine supracondyläre Drahtextension angelegt. 


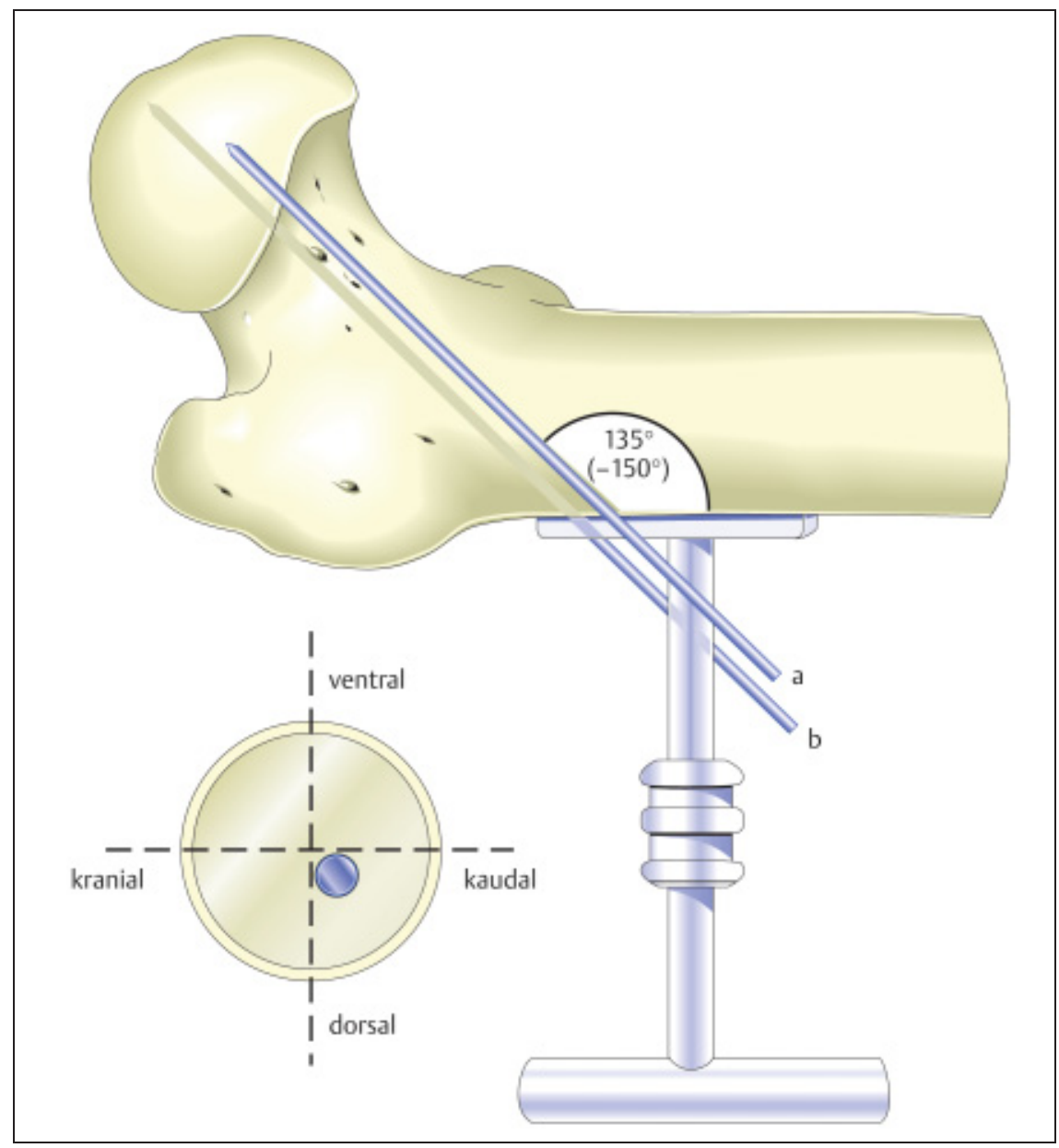

Abb. 8 Platzierung des Führungsdrahtes im dorsocaudalen Quadranten.

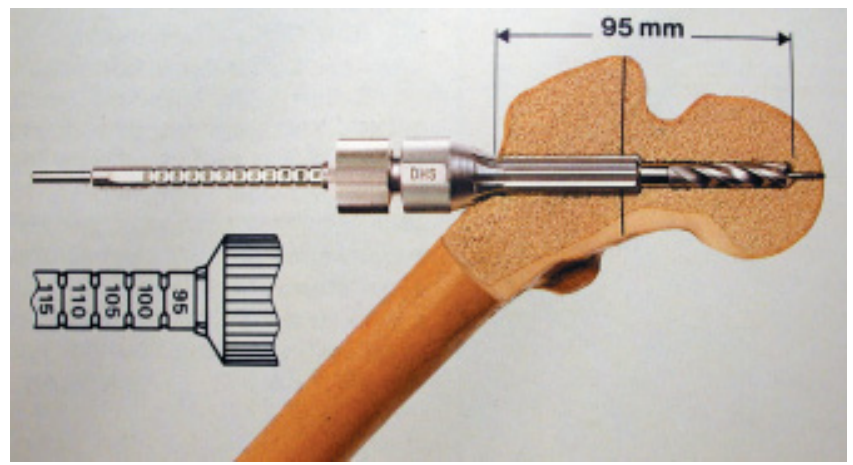

Ausmessen der Schraubenlänge mit dem Messgerät unter Abzug von $10 \mathrm{~mm}$. Einstellen des Dreistufenbohrers auf die ausgemessene Länge der DHS-Schraube.

Anschließend Überbohren des Führungsdrahtes mit dem Dreistufenbohrer (Abb.9). Der geschaffene Bohrkanal hat hierdurch den passenden Durchmesser für die Schraube im Femurkopf, im Schenkelhals für den Gleitzylinder an der Platte und an der lateralen Kortikalis
Abb. 9 Überbohrung des Führungsdrahtes mit dem 3-Stufen-Bohrer.

impacktiert. Die DHS-Platte wird mit mindestens drei $4,5 \mathrm{~mm}$ Kortikalisschrauben besetzt. Anschließend Komprimierung des Bruchspaltes mit der Kompressionsschraube, die danach wieder entfernt wird. Abschließende Bildwandlerkontrolle und radiologische Dokumentation (Abb.10a u. b).

Schichtweise Wundverschluß über einer Redon-Drainage und Lagern des Beines in einer Schaumstoffschiene.

Bei guter Knochenqualität kann durch die DHS-Sperrvorrichtung die Dynamisierungsfunktion der DHS-Schraube blockiert werden. Somit wird ein Zusammensintern und gleichzeitige Verkürzung des Beines verhindert.

Die Blockierung des Gleitvorganges der DHS ist jedoch nur bei guter Knochenqualität sinnvoll, da bei osteoporotischem Knochen ein Zusammensintern der Fraktur in einem gewissen Grade er-
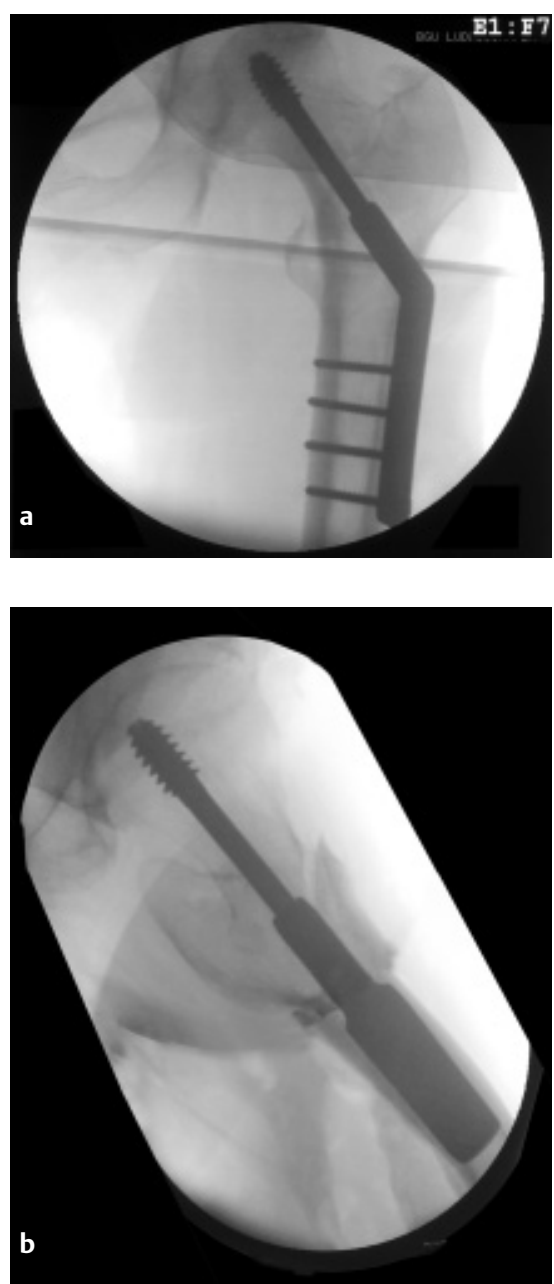

Dann wird die DHS-Platte, in der Regel 4Loch-Platte, in den vorgefrästen Kanal aufgesteckt und mit dem Einschlagbolzen

für den verdickten Übergang des Zylinders in die Platte.

Bei osteoporotischem Knochen kann auf das Gewindeschneiden verzichtet werden. Anschließend Eindrehen der DHSSchraube mit Zentrierhülse bis zur Nullmarke.

Abb.10a u. b Intraoperative Bildwandlerkontrolle und Dokumentation. 


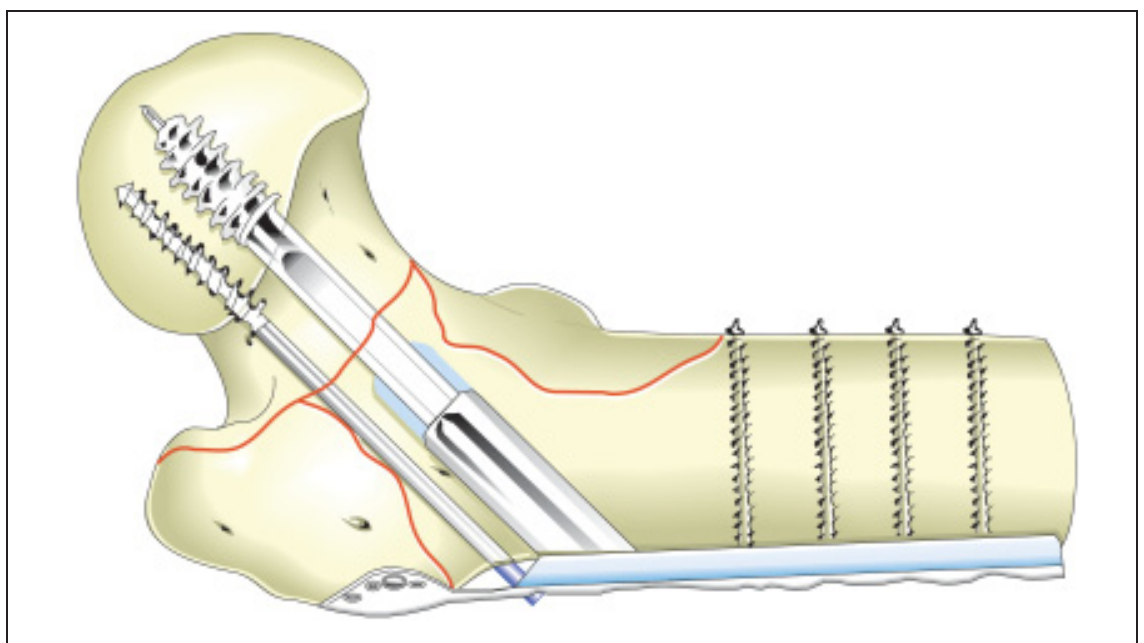

Abb.11 DHS mit Abstützplatte und Antirotationsschraube.

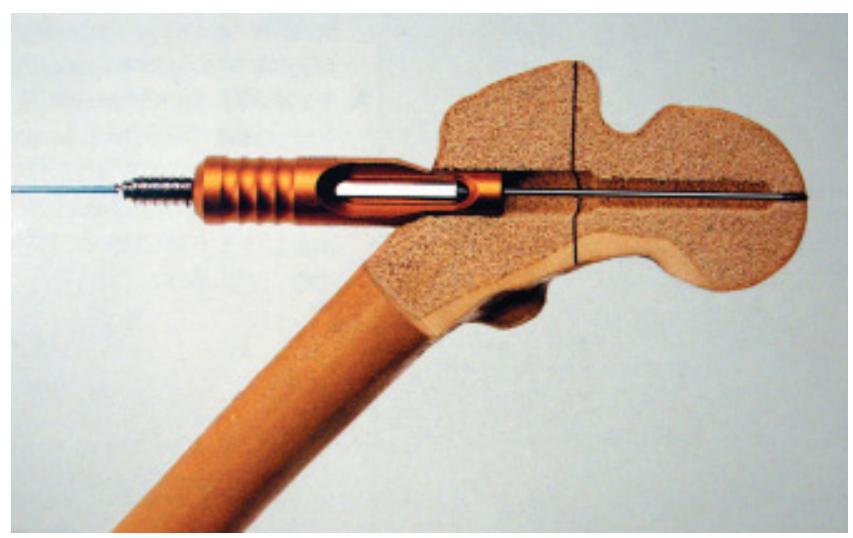

Abb.12 Neupositionierung eines verloren gegangenen Führungsdrahtes mittels Zentrierhülse.

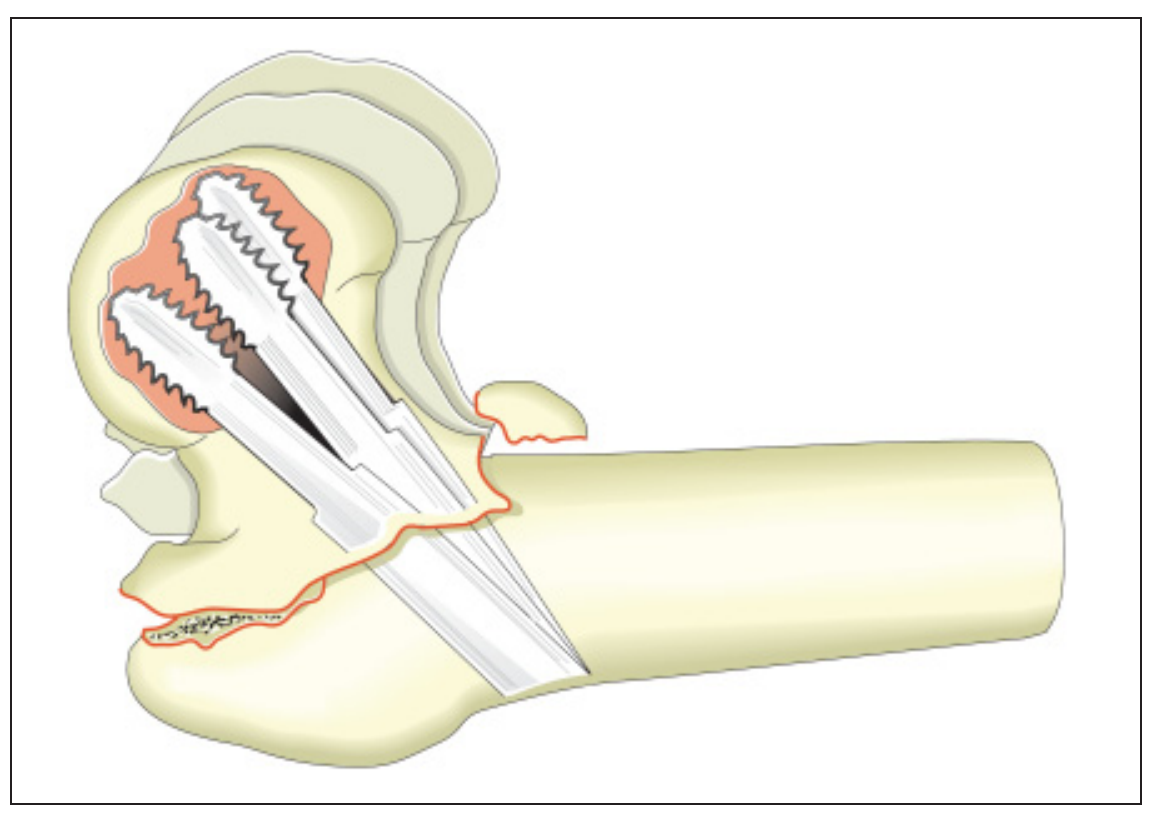

Abb.13 Defektzone im Femurkopf nach mehrfachen Bohrversuchen. wünscht ist um eine knöcherne Ausheilung positiv durch Erhöhung der Kompression zu unterstützen.

Fakultativ kann eine Antirotationsschraube zusätzlich über der DHS-Schraube eingebracht werden.

Bei großer Trümmerzone oder fehlender medialer Abstützung, insbesondere bei A2- und A3.3-Frakturen, kann zusätzlich eine Trochanterabstützplatte benutzt werden (Abb.11). Die DHS- Trochanterstabilisierungsplatte dient als Verlängerung der DHS-Platte zur Stabilisierung des Trochanter major. Sie verhindert, beim Zusammensintern der Fraktur, den Medialisationseffekt des Schaftes gegenüber dem Kopf/Hals Fragment.

Hier ist jedoch zu bedenken, dass hier Grenzindikationen für die Nutzung der DHS vorliegen und ein intramedulläres Verfahren geeigneter zur Versorgung dieser Frakturtypen ist.

\section{Tipps und Tricks}

Sicherstellen, dass nach Lagerung und Reposition eine korrekte Durchleuchtung ap und axial möglich ist. Erst dann sterile Abdeckung des OP-Gebietes und des Bildwandlers.

Sollte beim Aufbohren mit dem Dreistufenbohrer der Führungsdraht verloren gehen, unbedingt den Draht mit Hilfe der langen Zentrierhülse und einer umgedreht eingeführten DH Schraube wieder exakt zentrisch platzieren, da sonst Fehllagen der DHS-Schraube möglich sind (Abb.12).

\section{Fehler und Gefahren}

Häufigster Fehler ist die falsche Platzierung des Führungsdrahtes. Aufgrund der großdimensionierten DHS-Schraube ist ein mehrfaches Aufbohren mit dem Dreistufenbohrer nicht möglich (Abb.13).

Bei schlechter Knochenqualität, ungünstigem Frakturtyp und nicht optimaler Schraubenlage kann es zu einem „cutting out" der DHS-Schraube aus dem Femurkopf kommen.

Ebenso Folge schlechter Knochenqualität und mangelnder Abstützung ist übermäßiges Zusammensintern mit daraus resultierender Beinverkürzung .

Eine Hüftkopfnekrose ist bei Anwendung der DHS bei pertrochantären Frakturen 
eher selten, bei Verwendung der DHS bei der Schenkelhalsfraktur jedoch durch die unterschiedliche Blutversorgung häufiger.

Als salvage Verfahren kommen die $95^{\circ}$ Winkelplatte und die Endoprothese zum Einsatz.

\section{Postoperative Nachbehandlung}

Die Krankengymnastik mit Bewegungsübungen beginnt am ersten postoperativen Tag. Bei den A1- und A2-Frakturen ist in der Regel die Vollbelastung sofort möglich. Aufgrund der mangelnden medialen Abstützung ist bei den A3-Frakturen eine Teilbelastung von $20 \mathrm{~kg}$ über 6 Wochen zu empfehlen.

\section{Metallentfernung}

Beim alten Menschen ist eine Entfernung des Implantates in der Regel nicht sinnvoll. Es sei denn es kommt durch das übermäßige Zusammensintern der Fraktur zu einem Überstehen der DH Schraube aus dem Gleitzylinder der Platte und damit zu Irritationen der Weichteile über dem Trochanter.

Wegen des verbliebenen großen Defektes und der Möglichkeit einer Spontanfraktur am Schenkelhals, sollte beim jungen
Menschen die Metallentfernung durch eine Spongiosaplastik ergänzt werden.

\section{Ergebnisse}

Die Pseudoarthroserate liegt bei $4 \%$, die Rate der Kopfnekrose bei $2 \%$. Reeingriffe sind, je Literaturangabe, zwischen 2 und $11 \%$ notwendig.

\section{Zusammenfassung}

Obwohl die DHS in die Jahre gekommen ist, ist sie nach wie vor ein geeignetes und kostengünstiges Osteosyntheseverfahren zur Versorgung von A1-Frakturen. Die Indikation für A2- und A3-Frakturen hingegen ist kritisch zu sehen. Diese Frakturen sind unseres Erachtens durch ein intramedulläres Verfahren sicherer und belastungsstabiler zu versorgen, so dass hier die DHS ihren Stellenwert verloren hat.

\section{Literatur}

1 Aymar M. Dynamische Hüftschraube (DHS). In: Cotta, H., A. Wentzensen, F. Holz, K.-L. Krämer, J. Pfeil: Standardverfahren in der operativen Orthopädie und Unfallchirurgie. Thieme, Stuttgart 1996; 534-539

2 Bray TJ. Osteosynthese - Arbeitsbuch und Atlas. VCH, Weinheim 1993; 144-149

${ }^{3}$ Delee JC. Intertrochanteric Fractures. In: Rockwood, C.A.Jr., D.P. Green, R.W. Buchholz: Rockwood and Green's Fractures In Adults. 3rd Edition. J.B. Lippincott, Philadelphia $1991 ; 1538-1560$
4 Euler E, Krueger P, Schweiberer L. Die Behandlung hüftnaher Femurfrakturen mit der dynamischen Hüftschraube (DHS). Operat Orthop Traumatol 1990; 3: 203

5 Gruss M, Traut R. Die Versorgung instabiler pertrochantärer und per- bis subtrochantärer Oberschenkelbrüche mit der dynamischen Hüftschraube (DHS). Akt Traumatol 1992; 22: 144

${ }^{6}$ Mc Neill. Wis Med J 1975; 74: 129-130

7 Regazzoni P, Harder F. Die dynamische Hüftschraube. Operationstechnik, klinische Anwendung und Resultate. Chir Praxis 1985; 34: 51

8 Regazzoni P. The Dynamic Hip Screw (DHS) and Dynamic Condylar Screw (DCS). In: Müller, M.E., M. Allgöwer, R. Schneider, H. Willenegger: AO Manual Of Internal Fixation. 3rd Edition. Springer, Berlin 1991; $270-282$

9 Rütter A, Trentz O, Wagner M. Unfallchirurgie. Urban \& Schwarzenberg, München 1995; 664-665

Dr. med. Dietrich Schulte-Bockholt Unfallchirurg

Dr. med. Paul Alfred Grützner

Oberarzt

Dr. med. Alfred Schmidgen

Oberarzt

Prof. Dr. med. Andreas Wentzensen

Ärztlicher Direktor

BG-Unfallklinik Ludwigshafen der Universität Heidelberg

Ludwig-Guttmann-Str. 13

D-67071 Ludwigshafen 